\title{
PIE ROT
}

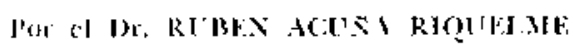

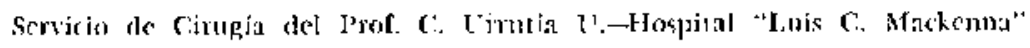

El presente trabajo tiene por objeto dar. cuenta, previo análisis somero del cuadro patológico, de la conducta asumida con les pies bot tratados en el Servicio de Cirugí: del Hospital Luis Calvo Mackenna, entre los años 1945-1950.

Se define el pie bot como todo aquel congénitamente deformado.

En este trabajo sólo nos referimos al pie bot varoequino supinado, que comprerde el $80-90 \%$ de todas las malformaciones congénitas pedias; ocupa el segundo lugar de las malformaciones congénitas (la luxación de la cadera ocupa el primer lugar), en un $7-8 \%$ se acompaña de otras malformaciones (luxación congénita de la cadera), es más frecuente en el hombre, $65 \%$, tiene tendencia a ser bilateral, $43 \%$ y representa el 1: 1000 de todos los recién nacidos.

Respecto a su etiología sólo enumeraremos las teorías que hay al respecto: deformación primitiva del esqueleto, suspensión del desarrollo, compresión uterina, disfunción neuromuscular, factor heredo-familia:, etc.

En lo que a pronóstico se refiere tiene especial importancia la precocidad del tratamiento. El tipo patogénico de los pies bot: fetales y embrionario, hace variar el pro. nóstico, siendo mucho más favorable en los del primer grupo. Lo mismo sucede con respecto a la forma del pie alterado: pie en muñón y pie esbelto y alargado. El primero caracterizado por el antepié corto y anclso, con el calcáneo alto y mal desarrollado, que muestra un equino visible, es de más mal pronóstico que aquel pie largo, estrecho y con antepié supinado y en adducción.

Sintomntología. Por la simple inspección se observa la triple actitud viciosa que pros. senta el pie bot: varismo, adducción del antepié, supinación, rotación hacia dentro de la planta del pie $y$ el equinismo, extensión exagerada del pie.

También se puedé apreciar mediante la simple observación una ligera atrofia mus- cular del pie y pierna (falta relieve pantorrilla) $y$ el predominio de los supinadores sobre Ios pronadores.

El plantigrama revela sólo la marca ex. terna del pie.

Si el enfermo deambula se exageran las, deformidades, acentuándose la rotación interna del pie (supinación calcáneo), de tal manera que el apoyo plantar se hace con el borde externo y aun con la cara dorsal del pie, mostrando en estos sitios callosidades, bolsas serosas, escaras, etc.

Por la exploración manual nos damos cuenta del grado de anormalidad del pie, de sus relaciones óseas, de la tensión de los tendones, de los tendones, de la existencin de una ęspina bífida, etc.

El estudio radiológico nos permite analizar el desarrollo del esqueleto del pie. Se sabe que los nódulos de osificación del escafoides $y$ de las cuñas sufren un gran rechazo y que la forma definitiva de los huesos del tarso es anormal. Pero cuando aparecen a su tiempo y sus relaciones son normales, la forma y la función de los diferentes huesos también es normal. Guntz ha dirho: Io esencial es la radiografía, que revela la deformidad del calcáneo y astrágalo. Esto tiene toda su importancia en los lactantes, en los que no ha intervenido la gravitación $\mathrm{y}$ en los cuales los huesos del tarso no están complemente formados. pues son en parte cartilaginosos.

Recordemos que en el lactante normai los ejes longitudinales del astrágalo y calcáneo forman un ángulo en el sentido dorso. plantar $y$ lateral, en canbio, en el niño con pie bot, estos ejes son paralelos en ambos sentidos.

Si consideramos un pie bot de grado moderado en el niño mayor (o adulto) veremos en la radiografía dorso-plantar: adducción del antepié y el escafoides articulado con la cara interna del astrágalo; en el sentido lateral el extremo posterior del calcáneo se ve elevado $\mathrm{y}$ el astrágalo en flexión plantar, siguiendo al calcánøo (equino). La 
vista posterior del pie revela gran supinación del calcáneo. Podría decirse que todos los huesos del pie giran 90 grados hacia afuera. Finalmente; se puede apreciar la rotación externa que sufre el extremo inferior de la piema, de tal manera que el peroné st. hace posterior.

Anatomía patológica. Difiere según se trate del lactante o del niño mayor. En al primero priman las alteraciones articulares, sobre todo a nivel de las articulaciones mediotarsianas (desviación antepié), subastragaliras (desviación del calcáneo) y tibiotarsianas (hiperextensión astrágalo). En el niño mayor, en cambio, predominan las lesiones óseas, las musculares y tendinosas. Jas alteraciones óseas se manifiestan por desplazamientos y deformaciones de los huesos, de grado variable, según la etiología y tiempo transcurrido de la enfermedad. Las consecuencias de estas alteraciones se hacen presentes a nivel de los huesos por descalcificación y menor crecimiento de algunos (escafoides, astrágalo) o por densificaciones de otros (calcáneo, cuboides) y también sobre las partes blandas, ligamentos y músculos: los primeros sufren alargamientos (los del dorso $y$ borde externo) o acortamientos (los plantares y del borde interno) y los ritúsculos, atróficos en general, pueden presentarse retraídos (tibial anterior y posterior, flexor común, triceps) o distendidos (peroneos laterales).

Tratamiento. No existe la curación espontánea, el pie bot debe tratarse.

El tratamiento tiende a corregir la deformidad y a mantenerla en la nueva posición. Mientras más precoz se hace, menor es la resistencia que oponen las partes blandas $y$ mayores las posibilidades que ofrecen después en su desarrollo normal la forma $y$ la función del pie. Sin embargo, hay que recordar que existen pocas afecciones con mayor tendencia a la recidiva que el pie bot. a pesar de los cuidados más solícitos y de los aparatos más correctos empleados en su tratamiento.

El pie varoeauino evoluciona progresivamente con la edad; esto hace que, refiriéndonos a su tratamiento. se puedan distinguir tres periodos:

El primero, de la reductibilidad completa, es aplicable a niños pequeños. uno a uno y medio años, en los cuales la reducción manual es suficiente y necesaria, siempre que su control sea mantenido durante años. Spit. zy refiere que mientras más temprano se lleva una deformidad congénita a la normalidad, tanto mejor será el resultado, ya que el niño crece con gran rapidez e irá creciendo, en este caso, hacia la forma normal y no hacia la anormalidad.

El tratamiento precoz es hoy una exigencia ortopédica general, respaldada por al parecer del especialista.

En el segundo período, de la irreductibilidad relativa, observada en niños poco mayores, más de un año, los huesos del pie no pueden recuperar sus relaciones normales, porque las diversas adaptaciones o retracciones (cápsulo-ligamentosas o musculares) que han sufrido, se 10 impiden. De modo que para facilitar o lograr la reducción necesaria se hace imprescindible la sección de estos diversos frenos.

El tercer período, llamado de la irreductibilidad absoluta, comprende Jos pies bot inveterados, los cuales sólo logran corregirlos las intervenciones quirúrgicas practicadas sobre el esqueleto.

El tratamiento propiamente tal puede ser ortopédico o incruento y quirúlgico. El primero, a su vez, comprende el tratamiento manual simple y el manual forzado.

La reducción manual simple que se usa ospecialmente en los lactantes y en general con buenos resultados, se vale de maniobras metódicas, suaves y progresivas, sin el uso de anestesia y su objeto es llevar al pie a la hipercorrección. Al efectuar las maniobras de reducción hay que considerar el asiento articular que tiene cada una de las deformaciones que dan la triple actitud viciosa del pie bot. Así el varo del antepié tiene su asiento en la articulación mediotarsiana, el varismo del calcáneo en la subastragalina y el equinismo a nivel de la articulación tíbiotarsiana. Wisbrun dice: primero que se distiendan las partes blandas internas $y$ plantares que están retraída; hasta que se consiga que el calcáneo, cuboides $y$ escafoides puedan ser llevados alrededor del astrágalo, siguiendo un movimiento de pronación $y$ abducción. Conseguido esto, se puede proseguir la corrección, restableciendo las condiciones normales en la articulación de Chopart, y luego, haciendo descender el calcáneo. Diríamos que primero se corrige la adducción del antepié, luego la supinación, para fínalizar con el equinismo.

El modelamiento incruento constituye lak vía lerapéutica ideal para curar los pies bot. Es el único método capaz de corregir de 
un modo uniforme los importantes defectos que presentan ligamentos, tendones $y$ músculos.

La corrección obtenida va seguida de un vendaje de yeso (que va desde el tercio medio del muslo hasta los ortejos, dejando la rodilla en flexión de 90 grados), que se mantiene durante un mes y sobre el cual se fractican las ventanas o tapas de corrección con una frecuencia de 4 a 8 días.

El tiempo empleado en la corrección completa del pie enfermo es sumamente yariable, diríamos que fluctúa entre 2 a 3 meses $y$ varios años. Conseguida dicha corrección debe seguirse por un largo período (varios años) con masajes, movimientos pasivos, empleo de plantilla, de valvas, etc., sudiéndose, incluso, completar dicha corrección, sea con tenotomlas (elongación del tendón de Aquiles en los casos de calcáneo alto) o con trasplantes tendinosos (tibial anterior al peroneo lateral corto, cuando hay tendencia a la supinación).

La reducción forzada que puede hacerse manualmente o por medio de aparatos llamados osteoclastos (Putti, Schultze, Still. Gocht, etc.) encuentra su mejor indicación en los pies bot inveterados (que no han sido tratados, o que no han sido tratados enérgica y concienzudarnente, por lo que presentan marcadas alteraciones articulares y óseas) y siempre necesita la acción de la anestesia general.

La reducción en estos casos se obtiene desgarrando ligamentos. aplastando huesos - produciendo desprendimientos epifisarios de la tibia o peroné. En otras palabras, estas correcciones violentas, por una parte dañan los huesos del tarso, que en gran parte están en su fase cartilaginosa $y$ por otra, hacen lo propio con la epífisis tibial, sobre la que se hinca la polea astragalina al realizar la inflexión dorsal forzada $\mathbf{E s}$ lógico, entonces, que no se pueda obtener una función satisfactoria con calcáneos, astrágalos y escafoides deformados, y por otra parte, los trastornos del crecimiento provocados en la epífisis inferior de la tibia hacen irreparable la posición viciosa en varo del antepié. Por eso es frecuente encontrar en los pies equinovaros, que han pasado por muchas manos, las huellas del tratamiento.

Las reducciones incruentas pueden o no ir asociadas a intervenciones quirúrgicas, hechas sobre las partes blandas o sobre éstas $\mathrm{y}$ el esqueleto, respectivamente. En el lactante y niños menores, en general, sólo se recurre a las intervenciones del primer tipo; en cambio, en los niños mayores y adolescentes "suele recurrirse a-alguna intervención ósea (resección en cuña. y."gr.), la que en todo caso no será muy extensa, por tratarse de pies ya corregidos anteriormenue. De este modo se ahorrará sustan‘ia. lográndose una forma del pie próxima a la normal, en especial a lo que dice relación cor su longitud. Así se consigue mejorar la forma del pie y aliviar sus moles. tias, aun cuando su función deja algo que desear. Las molestias pueden ser. escasas comparadas con la deformidad. debido a una extensa capacidad de adaptación funcional que tienen los niños mayores y más aún los aduitos.

El tratamiento quirúrgico o cruento dado por el fracaso del tratamiento ortopédico o por la edad del paciente (enfermos que vienen a tratarse tardíamente), puedo efectuarse sobre los elementos blandos solamente, sobre el esqueleto o comprender ambas partes a la vez.

Las operaciones que no afectan al esqueleto, sino exclusivamente a los tendones y al aparato cápsulo-ligamentoso (Codivilla, Gamboa), suelen dar resultados muy duraderos. Pero cuando estas intervenciones no scn suficientes se actúa sobre el esqueleto con operaciones que pueden ser de indole muy diversa. Existen numerosos -procedinuentos acreditados, que en la práctica conviene, a veces, combinar mutuamente (resecciones cuneiformes del medio pie y subastragalinas, llamadas doble o triple artrodesis).

De un modo general puede decirse que las intervenciones cruentas hechas sobre st esqueleto se aconsejan en aquellas lesiones marcadas $y$ groseras, que son operaciones mutiladoras y que pueden practicarse tanto en el tarso anterior como en el posterior o sobre ambos, respectivamente.

Finalmente, diremos que el pie bot no da una incapacidad absoluta, pero con frecuencia da molestias de consideración rcallos, bolsas serosas, inflamaciones, úlceras de decúbito, cansancio rápido, artritis, no permite usar zapato corriente, etc.). Los enfermos se sienten muy impresionados por la deformidad; por esto es absolutamente necesario, tanto desde el punto de vista médico como desde el punto de vista social, un tratamiento apropiado. 


\section{Resumen de la casuistica}

Se presentan 94 casos de pies bot atendidos entre los años 1945-1950 en el Servicio de Cirugía del Hospital Calvo Mackenna.

La edad de los nin̄os fluctuó entre el recién nacido (que empezó a tratarse al mes de edad, a fin de que la piel tolerara mejor el yeso) y 13 años.

En la distribución por sexos, $62(65,9 / 4)$ correspondieron a varones y $31(32,9 \% \mathrm{c})$ a mujeres.

En 40 niños la enfermedad era bilateral $\left(45,5^{\prime}\right)$, siendo en los 54 restantes sólo de un pie $(27$ del pie derecho y 27 del pie izquierdo).

En $68(72,3 \%)$ enfermitos sólo se había practicado tratamiento ortopédico, aun cuando hay 8 casos que pronto necesitarán de una intervención quirúrgica complementaria (trasplante del tibial anterior al peroneo lateral corto). Los $26(27,6 \%) \mathrm{ni}$ nos restantes fueron intervenidos quirúrgicamente, en 21 sobre las partes blandas (elongación del tendón de Aquiles, trasplantes tendinosos, Codivilla, Gamboa, etc.) y sólo en 5 sobre el esqueleto (doble y triple artodesis)

Descartando los 26 nin̄os operados sólo se usó anestesia en 16 pacientes de todos los sometidos a las correcciones sucesivas, seguidas de yeso.

Respecto a la duración del tratamiento el tiempo mínimo fué de 2-3 meses $y$ el mittximo de 4 años 5 meses.

Es importante hacer notar el hecho de que $51(54,2 \%)$ casos abandonaron, rechazaron el tratamiento o no siguieron controlándose después de haber estado corregida su deformación.

Finalmente, puede agregarse que de los 94 enfermos tratados, $23(24,4 \%(1)$, fuera de los que seguramente no lo declaran, habian iniciado su tratamiento en otros Servicios hospitalarios.

\section{Conclusiones}

1. El pie bot congénito tratado correcta y oportunamente, esto es, desde las primeras semanas de edad es susceptible de corregirse total, rápida y fácilmente.

2. Las manipulaciones correctoras deben ser suaves y gradualmente progresivas, venciendo primordialmente la adducción.

3. Después de haber hipercorregido la adducción y la supinación, debe tratarse el equinismo.
4. El procedimiento de aparatos enyesndos, practicado por manos expertas, consideramos que es el ideal. El yeso debe com. prender del tercio medio del muslo hasta los ortejos, dejando la rodilla en flexión d $\epsilon$. 90 .

5. En los pies bot inveterados, rebeldes, deben practicarse intervenciones quirúrgicas modelantes, adecuadas.

6. El tratamiento debe considerarse terminado cuando el niño tiene la normalidad deseada (en general después de varios años).

\section{Resumen}

Se presentan 94 casos de pie varoequino tratados durante 6 años, en niños cuya edad tluctúa entre el jecién nacido y 13 años.

En el $45,5^{\circ}:$ la enfermedad compromete ambos pies $y$ en la distribución por sexs $65,9 \%$ eran hombres.

De preferencia se hizo tratamiento oxtopédico, 72,3\% siguiendo la técnica de Kite.

Como el pie bot tiene gran tendencia recidivante, estos enfermos deben contrölarse varios años después de conseguida la normalidad deseada.

\section{Simmaty}

94 cases of varoequine foot are reported, which have been treated during six years in children, whose ages fluctuated between new borns and 13 years.

In $45.5 \%$, the desease was from both feet, and the distribution per sex was $65.9 \%$ of bovs.

Orthopedic treatment was preferably used, $72.3 \%$ following Kite's technique.

As club foot has a great recurring tendency, these patients should be kept under control several years after normalization.

BIHIOU,HAHA

C.AMPBELI'S.-Opetalive onthoperlies. J. \$. Spect Hugh smith.

I:RLACIHER,-Ortopedia Operatoria.

FALDINI, J.-Mantal de Ottoperia.

FOSTON, Cimigia plástica $v$ reparaclora.

IOHMANX, f:-Jic \& picma. Sus afeciones : su tratamichto.

IAMES, F. BRAIISIOORD.-Ratiologia de huesos v irtirulaciones.

KIRSC:HNER-NORM IN.,-Cirugia.

YT.J SON,-Surgery by J, H. Kitc.


sulgie onthoperligue

SC.I[INZ-BAENSCH-FRIEDL-Rontgen. Dingnóstico. 